\title{
Skyrmion Multi-Walls
}

\author{
J. Silva Lobo* and R. S. Ward ${ }^{\dagger}$ \\ Deparment of Mathematical Sciences, \\ Durham University, \\ Durham DH1 3LE
}

October 29, 2018

\begin{abstract}
Skyrmion walls are topologically-nontrivial solutions of the Skyrme system which are periodic in two spatial directions. We report numerical investigations which show that solutions representing parallel multi-walls exist. The most stable configuration is that of the square $N$-wall, which in the $N \rightarrow \infty$ limit becomes the cubicallysymmetric Skyrme crystal. There is also a solution resembling parallel hexagonal walls, but this is less stable.
\end{abstract}

PACS 11.27.+d, 11.10.Lm, 11.15.-q

*email address: j.i.silva-lobo@durham.ac.uk

†email address: richard.ward@durham.ac.uk 
The Skyrme system, originally introduced as a model of nucleons, is an archetypal $(3+1)$-dimensional classical field theory admitting topological soliton solutions. Much is known about various types of skyrmion solutions, for example: isolated skyrmions in $\mathbb{R}^{3}$, up to relatively high charge [1, 2, 3]; a triply-periodic 'Skyrme crystal' [4, 5, 6, 2, ; a doublyperiodic 'Skyrme domain wall' [7]; and various types of singly-periodic 'Skyrme chains' [8].

The purpose of this note is to investigate static $N$-wall solutions, ie. the $N>1$ generalization of the single-wall fields discussed in [7]. If one has two (or indeed $N$ ) well-separated parallel walls, then the force between them can be made attractive by a suitable relative orientation of the fields. So one expects there to be solutions representing $N$ walls bound together, although a priori the walls might merge together to form a single wall.

We investigate this by numerical minimization of the energy, and our main findings are as follows. There are two obvious shapes for a single wall, namely square and hexagonal, and it is known [7] that the latter has slightly lower energy than the former. If walls are allowed to attract, then they do not merge, but remain identifiable as separate parallel walls. There is a stable bound configuration representing two parallel hexagonal walls, but this is not the lowest-energy 2 -wall state. For $N \geq 2$, the lowest-energy state consists of $N$ parallel square walls (each one being a square array of half-skyrmions), and as $N \rightarrow \infty$ this approaches the skyrme crystal.

The energy density of a static $\mathrm{SU}(2)$-valued Skyrme field $U\left(x^{j}\right)$ on $\mathbb{R}^{3}$ is defined to be

$$
\mathcal{E}:=-\frac{1}{2} \operatorname{tr}\left(L_{i} L_{i}\right)-\frac{1}{16} \operatorname{tr}\left(\left[L_{i}, L_{j}\right]\left[L_{i}, L_{j}\right]\right),
$$

where $L_{i}=U^{-1} \partial U / \partial x^{i}$, and $x^{j}=(x, y, z)$ are the spatial coordinates. In what follows, let us write $U=\Phi_{4}+\mathrm{i} \Phi_{j} \sigma_{j}$, where $\sigma_{j}$ are the Pauli matrices, and the real 4 -vector field $\boldsymbol{\Phi}=\left(\Phi_{1}, \Phi_{2}, \Phi_{3}, \Phi_{4}\right)$ satisfies $\boldsymbol{\Phi} \cdot \boldsymbol{\Phi}=1$.

In this note, we deal with configurations which resemble $N$ walls or sheets, each parallel to the $x y$-plane: so the field is periodic in $x$ and $y$ (with periods $L_{x}$ and $L_{y}$ respectively), and satisfies the boundary condition

$$
\Phi_{4} \rightarrow \begin{cases}1 & \text { as } z \rightarrow-\infty \\ (-1)^{N} & \text { as } z \rightarrow \infty\end{cases}
$$

For $N=1$, and more generally for $N$ odd, one has a domain wall which separates two vacuum regions, where $\Phi_{4}=1$ and $\Phi_{4}=-1$ respectively; for $N$ even, one has the same vacuum on both sides of the multi-layered sheet. In the asymptotic region $|z| \gg 1$, the three fields $\Phi_{j}$ are small; and they satisfy the Laplace equation, since the energy density reduces 
to $\mathcal{E} \approx\left(\partial_{i} \Phi_{j}\right)^{2}$. Assuming (without loss of generality) that $L_{y} \geq L_{x}$, we see by separating variables that the leading behaviour as $|z| \rightarrow \infty$ is typically $\Phi_{j} \approx C \sin (\mu y) \exp (-\mu|z|)$, where $\mu=2 \pi / L_{y}$. In particular, the fields approach their asymptotic values exponentially quickly, with a scale determined by the larger of $L_{x}$ and $L_{y}$.

The topological charge $Q$ (over a single cell) is

$$
Q=\int_{T^{2} \times \mathbb{R}} \mathcal{Q} d x d y d z
$$

where

$$
\mathcal{Q}=\frac{1}{24 \pi^{2}} \varepsilon_{i j k} \operatorname{tr}\left(L_{i} L_{j} L_{k}\right)
$$

is the topological charge density. We claim that $Q$ is an integer. If $N$ is even, then (2) allows us to regard $\boldsymbol{\Phi}$ as being defined, for topological purposes, on $T^{2} \times S^{1}$; and then $Q$ equals the degree of $\boldsymbol{\Phi}$. If $N$ is odd, then it is not quite so obvious why $Q$ is an integer, but it follows from the theorem in the Appendix of [8]. The energy $E$ is defined to be

$$
E:=\frac{1}{12 \pi^{2}} \int_{T^{2} \times \mathbb{R}} \mathcal{E} d x d y d z
$$

and it satisfies the usual Faddeev bound $E \geq Q$.

In what follows, we describe $N$-wall configurations which were found by numerical minimization of the energy functional $E$. We used a first-order finite-difference scheme for $E$, with the spatial points $(x, y, z)$ being represented by a rectangular lattice having lattice spacing $h$, and we applied conjugate-gradient minimization. The lattice error in $E$ goes like $h^{2}$, and we extrapolated the finite- $h$ results for both $E$ and $Q$ to $h=0$. The extrapolated value of $Q$ then gives a measure of the remaining error, which for the situations described below turns out to be less than $0.2 \%$. The boundary condition (2) was modelled by imposing $\Phi_{4}=1$ at $z=-L_{z} / 2$ and $\Phi_{4}=(-1)^{N}$ at $z=L_{z} / 2$. As remarked above, the walls are exponentially localized in $z$, and so as long as $L_{z}$ is taken to be large enough, there is no discernable finite-size effect; a value of $L_{z}=10+2 N$ turns out to be sufficient for this. In each case, we adjusted the periods $L_{x}$ and $L_{y}$ to their optimal size, meaning that the energy-per-cell is made as small as possible. Numerical minima were randomly perturbed and then re-minimized, as a test of their stability. As initial configurations we used the same sort of 'rational map ansatz' as in [7], involving a Weierstrass elliptic function of $x+\mathrm{i} y$ (the lemniscatic form to get square symmetry, and the equianharmonic form to get hexagonal symmetry), together with a suitable profile function $f(z)$ satisfying $f\left(-L_{z} / 2\right)=0$ and $f\left(L_{z} / 2\right)=N \pi$. 


\begin{tabular}{|c|c|c|}
\hline$N$ & $\widehat{E}$ & $L$ \\
\hline 1 & 1.068 & 4.25 \\
2 & 1.053 & 4.47 \\
3 & 1.048 & 4.54 \\
4 & 1.046 & 4.58 \\
5 & 1.044 & 4.61 \\
\hline
\end{tabular}

Table 1: Energy $\widehat{E}$ and cell-size $L$ for the square $N$-wall

The results are consistent with the anticipated general principle that the lowest-energy configurations are arrays of half-skyrmions. For an $N$-wall, we expect that each fundamental cell will contain a multiple of $4 N$ half-skyrmions, and therefore its topological charge $Q$ will be a multiple of $2 N$; this indeed turns out to be the case. As mentioned above, the walls do not merge, but retain their identity; the location of each wall can be determined by looking at the locus where $\Phi_{4}=0$.

The simplest case to describe is the square one, with $L_{y}=L_{x}=L$; our results for $1 \leq N \leq 5$ are summarized in Table 1 , which gives the energy-per-charge $\widehat{E}$, and the optimal value of $L$, for each $N$. Pictures of the $N=1$ and $N=2$ cases are presented in Figure 1, together with a plot of the energy data in Table 1. Let us first comment on the data. The normalized energy $\widehat{E}$ of the square $N$-wall is surprisingly close to having a $1 / N$ dependence (although there is no obvious reason why this should be so); and extrapolating on this basis gives $\widehat{E} \approx 1.039$ in the $N \rightarrow \infty$ limit. This is very close to the energy of the (triply-periodic) skyrme crystal, a cubic array in which each fundamental cube contains eight half-skyrmions: its energy-per-charge, computed using the method described above, is $\widehat{E}=1.038$. Further support for the claim that the square $N$-wall tends to the skyrme crystal as $N \rightarrow \infty$ comes from looking at the symmetries of the field $\boldsymbol{\Phi}$. These include, for example, the translations

$$
\begin{aligned}
x \mapsto x+\frac{1}{2} L_{x} & \Rightarrow \quad\left(\Phi_{1}, \Phi_{2}, \Phi_{3}, \Phi_{4}\right) \mapsto\left(-\Phi_{1},-\Phi_{2}, \Phi_{3}, \Phi_{4}\right), \\
y \mapsto y+\frac{1}{2} L_{y} & \Rightarrow \quad\left(\Phi_{1}, \Phi_{2}, \Phi_{3}, \Phi_{4}\right) \mapsto\left(\Phi_{1},-\Phi_{2},-\Phi_{3}, \Phi_{4}\right), \\
z_{p} \mapsto z_{p+1} & \Rightarrow \quad\left(\Phi_{1}, \Phi_{2}, \Phi_{3}, \Phi_{4}\right) \mapsto\left(\Phi_{1},-\Phi_{2}, \Phi_{3},-\Phi_{4}\right),
\end{aligned}
$$

where the third translation (in $z$ ) denotes moving from the $p^{\text {th }}$ wall to the $(p+1)^{\text {st }}$ wall. These are exactly the same as the translation symmetries of the skyrme crystal [2]. The values for the optimal cell-length $L=L_{x}=L_{y}$ are consistent with their approaching $L=4.7$ as $N \rightarrow \infty$, this being the cell-size of the skyrme crystal (and similarly the 
distance between each parallel pair of walls is approximately $4.7 / 2$, as one would expect).

Each 3 -dimensional plot in Figure 1 is an isosurface of the energy density $\mathcal{E}$, namely where $\mathcal{E}$ equals 0.6 times its maximum value. For the square case, the plots are over four fundamental cells. One clearly sees square arrays of half-skyrmions. Observe that, for $N=2$, the half-skyrmions are aligned in the $z$-direction; the same is true for $N>2$.

Let us turn now to the case of hexagonal symmetry. For ease of computation, we follow the same scheme as in [7], namely taking $L_{y}=\sqrt{3} L_{x}$ and fitting two fundamental parallelograms into the corresponding rectangle. Each such rectangle, of each wall, contains eight half-skyrmions, as is seen in the hexagonal 2-wall picture of Figure 1. For $N=1$, the energy of the hexagonal arrangement is $\widehat{E} \approx 1.062$, less than that of the corresponding square case [7]; but for $N \geq 2$, the hexagonal arrangement is less efficient than the square one, and (depending on the values of $L_{x}$ and $L_{y}$ ) it is either a local minimum of the energy functional, or it is unstable. There is a local-minimum hexagonal 2-wall solution with energy $\widehat{E} \approx 1.055$, which is only very slightly (less than $0.2 \%$ ) higher than that of the square 2-wall. Its energy density is depicted in Figure 1; one feature to note is that the two walls are not aligned in the $z$-direction, but are offset. If $L_{x}$ and $L_{y}$ are allowed to change so that the relation $L_{y}=\sqrt{3} L_{x}$ no longer holds, then this solution becomes unstable, and changes into the square 2-wall.

An isolated skyrmion of charge $Q \geq 3$ typically has a polyhedral-shell structure, analogous to carbon fullerenes, and it may be viewed as constructed from a section of the hexagonal 1-wall (graphene), with the insertion of defects to create a spherical shell [7, 2]. There has also been an investigation [9] of the possibility of constructing skyrmions as multi-walled spherical shells, with the 'shell material' consisting of a double or triple wall. For the cases that were examined in [9], either the walls coalesced, or one obtained a structure which resembled a shell-like part of the skyrme crystal. The findings reported above are consistent with this; in particular, multiple hexagonal walls appear to be rather unstable, and therefore unsuitable for constructing shells. But it does raise the possibility of stable high-charge skyrmions constructed as shells of square multi-wall material, or equivalently as hollow chunks of skyrme crystal, and this would be worth investigating further.

Acknowledgment. Support from the UK Science and Technology Facilities Council, through the Rolling Grant 'Particles, Fields and Spacetime', is gratefully acknowledged. 


\section{References}

[1] R Battye and P M Sutcliffe, Skyrmions, fullerenes and rational maps. Rev Math Phys 14 (2002) 29-85.

[2] N S Manton and P M Sutcliffe, Topological Solitons. (Cambridge University Press, 2004)

[3] R Battye, N S Manton and P M Sutcliffe, Skyrmions and the $\alpha$-particle model of nuclei. Proc Roy Soc Lond A 463 (2007) 261-279.

[4] I Klebanov, Nuclear matter in the Skyrme model. Nucl Phys B 262 (1985) 133-143.

[5] M Kugler and S Shtrikman, A new Skyrmion crystal. Phys Lett B 208 (1988) 491-494.

[6] L Castillejo, P S J Jones, A D Jackson, J J M Verbaarschot and A Jackson, Dense Skyrmion systems Nucl Phys A 501 (1989) 801-812.

[7] R Battye and P M Sutcliffe, A Skyrme lattice with hexagonal symmetry. Phys Lett B 416 (1998) 385-391.

[8] D Harland and R S Ward, Chains of skyrmions. JHEP 12 (2008) 093.

[9] N S Manton and B M A G Piette, Understanding skyrmions using rational maps. Prog Math 201 (2001) 469-480. 
$N=1$ square

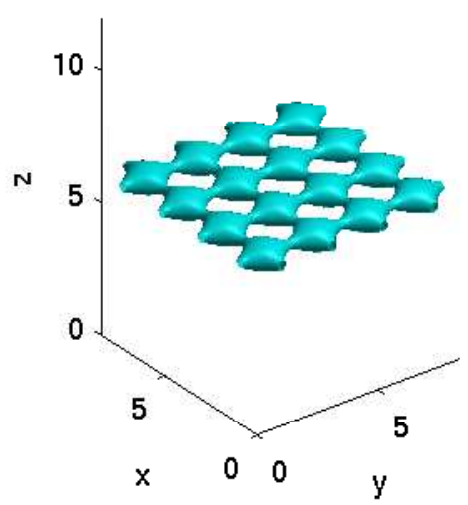

$\mathrm{N}=2$ hexagonal

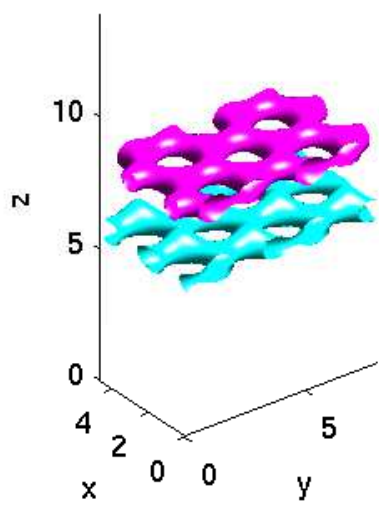

$N=2$ square
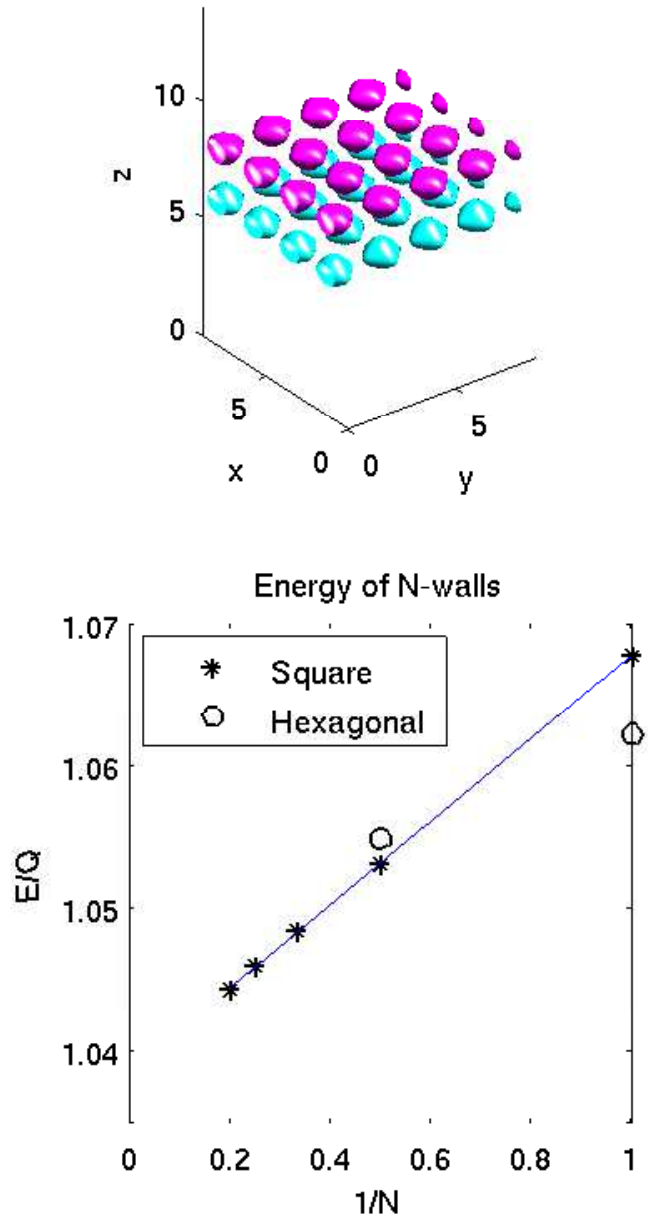

Figure 1: Energy densities of the square 1-wall, square 2-wall, and hexagonal 2-wall; and plot of the energy $\widehat{E}$ for the square $N$-wall $(1 \leq N \leq 5)$ and hexagonal $N$-wall $(1 \leq N \leq 2)$. 